\title{
Ginsenoside Rg3 Bile Salt-Phosphatidylcholine-Based Mixed Micelles: Design, Characterization, and Evaluation
}

\author{
Xiu Yu, ${ }^{a}$ Huan $\mathrm{Xu},{ }^{*, a}$ Meina Hu, ${ }^{a}$ Xiaojiao Luan, ${ }^{a}$ Kaiqian Wang, ${ }^{b}$ Yuanshan Fu, ${ }^{c}$ Di Zhang, ${ }^{a}$ and \\ Jianying $\mathrm{Li}^{a}$ \\ ${ }^{a}$ College of Chemistry and Chemical Engineering, Liaoning Normal University; Dalian 116029, People's Republic \\ of China: ${ }^{b}$ Dalian Fusheng Natural Medicine Development Company, Ltd.; Dalian 116600, People's Republic of \\ China: and ${ }^{c}$ Department of Anatomy, College of Basic Medical Science, Dalian Medical University; Dalian 116044, \\ People's Republic of China.
}

Received January 16, 2015; accepted March 3, 2015

20(R)-Ginsenoside $\operatorname{Rg} 3$ (G-Rg3) has good inhibition of tumor angiogenesis and anti-tumor effect. However, its poor aqueous solubility and liposolubility are not ideal for clinical applications. In this study, a G-Rg3 bile salt-phosphatidylcholine-based mixed micelle system (BS-PC-MMS) was prepared. The optimization of G-Rg3 BS-PC-MMS was carried out using response surface methodology based on a central composite design. The encapsulation efficiency (EE) and light transmission (LT) of the optimized formulation were $\mathbf{9 0 . 6 9 \pm 2 . 5 4 \%}$ and $99.10 \pm 3.12 \%$, respectively. The average particle size of micelles was $20 \mathrm{~nm}$. To increase the stability of G-Rg3 BS-PC-MMS, the lyophilized formulation of micelles was prepared. The G-Rg3 BS-PCMMS did not produce hemolysis of erythrocytes within a certain concentration range and exhibited a good inhibition of tumor cells. The chick embryo chorioallantoic membrane assay results showed that the G-Rg3 BS-PC-MMS significantly inhibited angiogenesis. The G-Rg3 BS-PC-MMS is thus shown to be a safe, stable, and promising drug delivery system.

Key words ginsenoside Rg3; lyophilized; hemolysis; bile salt-phosphatidylcholine-based mixed micelle; angiogenesis

20(R)-Ginsenoside $\operatorname{Rg} 3(\mathrm{G}-\mathrm{Rg} 3)$ is a tetracyclic triterpenoid saponin compound extracted from Chinese herbal medicine ginseng (Panax ginseng, C.A. MEY). The molecular formula and molecular weight of G-Rg3 are $\mathrm{C}_{42} \mathrm{H}_{72} \mathrm{O}_{13}$ and 784.30, respectively. The chemical name of $\mathrm{G}-\mathrm{Rg} 3$ is $20(R)$-dammarane alkenediol-3- $O-\beta$-D-glucopyranosyl group $(1 \rightarrow 2)-\beta$-D-glucopyranose. ${ }^{1,2}$ Many studies have demonstrated that G-Rg3 has an anti-proliferative effect in various cancers, including prostate cancer, lung cancer, stomach cancer, and liver cancer. ${ }^{3-9)}$ The target of its anti-tumor effect is the angiogenic vessels of tumor. Abnormal angiogenesis is an important step in the development of solid tumors. ${ }^{10,11)}$ Therefore, anti-angiogenesis is an important means of controlling tumor growth. ${ }^{12,13)}$ The extraction rate of G-Rg3 is only $0.003 \%$, and its efficacy is much higher than that of ginseng.

Unfortunately, G-Rg3 is almost insoluble in water. ${ }^{14)}$ It can slightly dissolve in the mixed solvent of chloroform and methanol and dimethyl sulfoxide (DMSO) and other organic solvents. Currently, the main dosage form of G-Rg3 is capsules. Its water and lipid insoluble nature greatly limits its clinical application. The administration of G-Rg3 has the following defects: (i) the short half-life of G-Rg3 allows it to be metabolized before reaching the target site ${ }^{15,16}$; (ii) the poor targeting of G-Rg3 distributes it in the normal tissues and organs aside from the tumor; and (iii) the low solubility of G-Rg3 in water may require the addition of a special co-solvent, which may cause many adverse reactions. To extend the half-life of the drug, improve its solubility, and enhance its function in the body, a new type of drug carrier for G-Rg3 should be created. In recent years, G-Rg3 has been designed as emulsions, microspheres, liposomes, and other formulations. ${ }^{17-20)} \mathrm{Com}-$ pared with other dosage forms, one of the most significant advantages of micelles is their very small particle size, which is generally less than $50 \mathrm{~nm}$. Micelles can penetrate the defective tumor vascular endothelium in tumor tissues with the help of enhanced permeability and retention. In this study, G-Rg3 was encapsulated in bile salt and phospholipids (PLs) mixed micelles to improve the solubility and tumor targeting of the drug.

Phospholipids are amphoteric compounds that contain the special structure of a quaternary ammonium salt, and have both hydrophilicity and lipophilicity. The main components of bile salt are cholalic acid and bile. Bile in the human body is abundant in bile salts and PLs, and it has an important function in the absorption of lipids, fat-soluble vitamins, and drugs. Bile salt is also referred to as a "physiological detergent" because the structure contains a hydrophilic hydroxyl group, carboxyl group, and hydrophobic methyl group. ${ }^{21,22)}$ Bile salt has surface activity and can reduce the surface tension between the lipid-water two-phase region. The bile salt-phosphatidylcholine-based mixed micelle system (BSPC-MMS) has been widely used for the delivery of insoluble drugs, such as paclitaxel, diazepam, and lorazepam. ${ }^{23-28)}$ The BS-PC-MMS not only can increase the solubility and encapsulation efficiency of poorly soluble drugs, ${ }^{23)}$ but it can also improve the bioavailability of these drugs. ${ }^{26)}$

In this study, the formulation of G-Rg3 BS-PC-MMS was optimized by response surface methodology (RSM) based on central composite design (CCD). ${ }^{29,30)}$ The physicochemical properties, stability, safety of administration, inhibition of tumor cells, and anti-angiogenesis effect of micelles were characterized and evaluated. This study provides a reference for the preparation of ideal nano drug delivery systems (DDS) of G-Rg3. 


\section{Experimental}

Materials G-Rg3 was provided by Dalian Fusheng Natural Medicine Development Co., Ltd. (Dalian, China) (Degree of purity >98\%; Lot No. 20130107). PL-98T Egg yolk phosphatidylcholine (EPC) was purchased from Shanghai Advanced Vehicle Technology Co., Ltd. (Shanghai, China). Sodium deoxycholate (SDC) was obtained from Beijing Aoboxing Biotechology Co., Ltd. (Beijing, China). Trehalose was obtained from Nanning Zhongnuo Biotechology Co., Ltd. (Nanjing, China). HPLC-grade acetonitrile and methanol for HPLC analysis were provided by Sigma-Aldrich (U.S.A.). 3-(4,5-Dimethylthiazol-2-yl)-2,5-diphenyltetrazolium bromide (MTT) was provided by AMRESCO (U.S.A.). Dextran gel G-50 of 50-100 $\mu \mathrm{m}$ was purchased from Amresco Biosharp (Solon, U.S.A.). The human melanoma cell line A375 was purchased from the cell bank of Chinese Academy of Sciences (Kunming, China). All other reagents and buffer solution components were analytical-grade preparations. Distilled and deionized water produced from Milli-Q Synthesis was used in all experiments. Phosphate buffer saline (PBS) buffer was prepared in the laboratory, and adjusted to a predetermined $\mathrm{pH}$ using a $\mathrm{pH}$ meter (Model PHS-25pH).

Preparation of G-Rg3 BS-PC-MMS BS-PC-MMS were prepared using the film dispersion method. Prescribed amounts of SDC and EPC were dissolved in a mixture of methanol-chloroform $(3: 2, \mathrm{v} / \mathrm{v})$ containing G-Rg3. The mixture was sonicated for $3 \mathrm{~min}$ to clarify. The solution was evaporated dry to form the film at $40^{\circ} \mathrm{C}$ until a constant weight was reached. The resulting films were dispersed in a given amount of dispersion medium (phosphate buffer, $0.067 \mathrm{M}, \mathrm{pH}$ 7.4). The suspension was centrifuged at $1500 \times \boldsymbol{g}$ for $15 \mathrm{~min}$ to remove the precipitate. Finally, the supernatant was passed through $0.15 \mu \mathrm{m}$ polycarbonate membranes to obtain clear micellar solutions with the required concentrations.

Optimized Preparation of G-Rg3 BS-PC-MMS by RSM Based on CCD Based on the preliminary experiments, two variables, namely, the molar ratio of EPC and total amount of accessories [EPC/(EPC+SDC) (molar ratio) (A)] and total concentration of excipient (B), were selected as the investigation factors to optimize the prescription of $\mathrm{G}-\mathrm{Rg} 3$ micelles. The selected factors were subjected to analysis by RSM with a two-factor-five-level system-CCD. The factor level settings are presented in Table 1. The encapsulation efficiency (EE) (R1) and light transmittance (LT) (R2) were used as indices to evaluate the prepared batches. The relationship between each factor and index was fitted, and the regression coefficients and constants were calculated using data processing software Design-Expert trial version 8.0.6 (Stat-Ease Inc., Minneapolis, $\mathrm{MN}$, U.S.A.). In addition, the accuracy and fitness of the regression formula were evaluated by the correlation coefficient. The response surfaces that described the relationship between each factor and index were drawn according to the fitting equation. Finally, the suitability of the model equation for predicting the optimum response values was tested using the recommended optimum conditions.

Stability of G-Rg3 BS-PC-MMS G-Rg3 BS-PC-MMS were placed at a $4^{\circ} \mathrm{C}$ environment for $7 \mathrm{~d}$. The EE and LT of G-Rg3 micelles were determined at different times.

Lyophilization of G-Rg3 BS-PC-MMS Trehalose was selected as the freeze-drying protective agent. The ratio of trehalose and PLs was 4:1 (w/w). The micellar solution was quickly frozen for $5 \mathrm{~h}$ and vacuum-dried for $30 \mathrm{~h}$ using a LGJ25 freezing dryer (Sihuan Scientific Instrument, Beijing, China), and the lyophilized preparation of G-Rg3 BS-PC-MM was obtained.

\section{Characterization of G-Rg3 BS-PC-MMS}

\section{Morphology of G-Rg3 BS-PC-MMS}

The morphology of BS-PC-MMS before lyophilization was observed using a transmission electronic microscope (TEM; JEM-2000EX, JEOL, Japan). The samples were diluted 10 times with phosphate buffer ( $\mathrm{pH}$ 7.4), placed on a copper grid coated with carbon film, and air-dried for $3 \mathrm{~min}$. The samples were then stained with $2 \%$ phosphotungstic acid for $3 \mathrm{~min}$. Finally, the samples were air-dried prior to TEM analysis.

A scanning electron microscope (SEM; SSX-500, Shimadzu, Japan) was used to obtain the SEM micrographs of the lyophilized products. A small amount of G-Rg3 BS-PC-MM was adhered to one side of double-faced adhesive tape. The surface float or excess freeze-dried samples were blown away. The other side of the double-faced adhesive tape was adhered to the sample holder. The samples were sprayed with gold by an ion beam sputtering apparatus. Finally, the samples were observed by SEM.

EE and LT of G-Rg3 BS-PC-MMS The EE of G-Rg3 BS-PC-MM was assayed by Sephadex microcolumn centrifugal-HPLC analysis. After the centrifugation of G-Rg3 micelles by the Sephadex microcolumn, the filtrate was collected and diluted by methanol. The same amount of drug-loaded micelles was diluted directly by methanol. The content of G-Rg3 in micelles was analyzed by HPLC. The chromatographic conditions of LC-100 HPLC (Shanghai Wufeng Scientific Instrument Co., Ltd.) were as follows: mobile phase, acetonitrile-water $(45: 55, \mathrm{v} / \mathrm{v})$; detection wavelength, $203 \mathrm{~nm}$; flow velocity, $1 \mathrm{~mL} \cdot \mathrm{min}^{-1}$. The EE was calculated by the following equation:

$$
\mathrm{EE} \%=\left(C_{\text {cen }} / C_{\text {tot }}\right) \times 100 \%
$$

where $C_{\text {tot }}$ is the total concentration of G-Rg3 in micelle suspensions, and $C_{\text {cen }}$ is the concentration of $\mathrm{G}-\mathrm{Rg} 3$ in centrifugal liquid.

The LT of micelles (without dilution) was quantified using a model 752N UV-Vis spectrophotometer (Shanghai Precision \& Scientific Instrument Co., Ltd., China) at a wavelength of $660 \mathrm{~nm}$.

Size Distribution and Zeta Potential of G-Rg3 BS-PCMMS The mean particle size and zeta potential of G-Rg3

Table 1. Factors and Levels of Central Composite Design

\begin{tabular}{lccccc}
\hline \hline \multirow{2}{*}{ Variable } & \multicolumn{4}{c}{ Levels } \\
\cline { 2 - 6 } & -1.414 & -1.00 & 0.00 & 1.00 & 1.414 \\
\hline A (EPC/EPC+SDC, w/w) & 0.26 & 0.30 & 0.40 & 0.50 & 0.54 \\
B (Total concentration of excipients, \%) & 0.59 & 1.00 & 2.00 & 3.00 & 3.41 \\
\hline
\end{tabular}


micelles were determined by a Zeta Potential/Particle Sizer (NICOMP 380 ZLS, U.S.A.) at a fixed angle of $90^{\circ}$. All measurements were conducted at $25^{\circ} \mathrm{C}$ in triplicates.

In Vitro Hemolysis Test Two percent erythrocyte suspension was incubated with saline (negative control group, NG), distilled water (positive control group, PG), and different volumes of micelles (experimental group, EG) at $37^{\circ} \mathrm{C}$ for $1 \mathrm{~h}$. The samples were centrifuged at $2000 \times \boldsymbol{g}$ for $10 \mathrm{~min}$, and the absorbance of the supernatants was measured at $565 \mathrm{~nm}$ by UV-Vis spectrophotometry (752N UV-Vis spectrophotometry, Shanghai, China). The hemolysis rate (HR) was calculated according to the following equation:

$$
\text { Hemolysis percent }(\%)=\left(A_{\mathrm{EG}}-A_{\mathrm{NG}}\right) /\left(A_{\mathrm{PG}}-A_{\mathrm{NG}}\right) \times 100 \%
$$

where $A_{\mathrm{EG}}, A_{\mathrm{NG}}$, and $A_{\mathrm{PG}}$ are the absorbance of EG, NG, and $\mathrm{PG}$, respectively.

Another group of samples was incubated with different solutions (saline, distilled water, and micelles) at $37^{\circ} \mathrm{C}$ for $3 \mathrm{~h}$, and the degree of hemolysis was observed using the naked eye.

Tumor Cell Inhibition Assay The cytotoxicity of G-Rg3 BS-PC-MM was evaluated by MTT assay in A375 cell lines. Cells were seeded into 96-well microtiter plates at a density of $5 \times 10^{3}$ cells per well in Dulbecco's modified Eagle's medium supplemented with $10 \%$ fetal bovine serum at $37^{\circ} \mathrm{C}$ in an atmosphere of $5 \% \mathrm{CO}_{2}$ and $90 \%$ relative humidity. The culture medium was then replaced with fresh medium. Cells were treated with aqueous solutions of the free drug and micelles at increasing G-Rg3 concentrations of 50, 70, 90, 110, 130 and $150 \mu \mathrm{g} \cdot \mathrm{mL}^{-1}$. The $\mathrm{NG}$ group was treated with normal culture medium. After $48 \mathrm{~h}$ of incubation, the effects of different treatments on cell viability were assessed by MTT assay. Typically, $5 \mathrm{mg} \cdot \mathrm{mL}^{-1} \mathrm{MTT}$ in PBS was added to each well and incubated for $4 \mathrm{~h}$. The supernatants were removed, and the formazan crystals were dissolved in $150 \mu \mathrm{L}$ of DMSO. Aliquots were drawn from each well, and their optical density (OD) values at $492 \mathrm{~nm}$ were determined by a microplate reader (Model 680, BIO-RAD, U.S.A.). Cell viability at each concentration was calculated according to the following equation:

$$
\text { Cell viability }(\%)=100 \%-\mathrm{OD}_{\mathrm{D}} / \mathrm{OD}_{\mathrm{C}} \times 100 \%
$$

where $\mathrm{OD}_{\mathrm{D}}$ is the absorbance of the dosing group, and $\mathrm{OD}_{\mathrm{C}}$ is the absorbance of the control group.

Chick Embryo Chorioallantoic Membrane (CAM) Assay A window (about $1 \mathrm{~cm}$ in diameter) was cut in a fertilized 7-dold eggshell by a gimlet, and the embryo's head was found using a light source. Simultaneously, a small hole was made on the top of the air chamber of the chick embryo, and the chamber was sucked by a suction bulb. The shell membrane was carefully removed, and the CAM was exposed. Drugsoaked filter papers of different concentrations were gently placed on the CAM. The eggs were randomly divided into three groups: blank control, 0.1\% DMSO; and dosing groups, 0.1 and $0.5 \mathrm{~mm}$ G-Rg3 micelles. Finally, the gap was resealed with transparent film, and incubated at $37^{\circ} \mathrm{C}$ and $40-60 \%$ humidity for $3 \mathrm{~d}$. The aforementioned operations were carried out within a clean bench. The chicken embryo was partially fixed with acetone and ethanol for $10 \mathrm{~min}$ after $3 \mathrm{~d}$. The discharged filter paper on the CAM was cut, and the filter paper was removed. The growth of blood vessels was observed under a dissecting microscope, and photographs were taken using a digital camera (A590, Canon, Japan).

Statistical Analysis Statistical comparisons were performed by Student's $t$-test for two groups, and one-way ANOVA for multiple groups. A $p<0.05$ indicated statistical significance.

\section{Results and Discussion}

Optimization of the Prescription of G-Rg3 BS-PC-MMS A total of 13 experiments were run to optimize the two individual parameters by RSM based on CCD. The experimental conditions, EE, and LT of G-Rg3 BS-PC-MM according to the factorial design are shown in Table 2. The statistical significance of the regression model was checked by $F$-test. The ANOVA results show that the total model was highly significant $(p<0.05)$, and the "lack of fits" of EE $(p=0.3737)$ and LT ( $p=0.1383$ ) were insignificant. Thus, the model was sufficient and could fit well for the prediction of results.

After statistical analysis and fitting, multiple second-order equations were obtained as follows:

The final equation in terms of coded factors:

$$
\begin{aligned}
& \mathrm{EE}(\mathrm{R} 1)=90.74+0.64 \mathrm{~A}+0.19 \mathrm{~B}-2.53 \mathrm{AB}-3.55 \mathrm{~A}^{2}-1.17 \mathrm{~B}^{2} \\
& \mathrm{LT}(\mathrm{R} 2)=99.14+0.41 \mathrm{~A}+0.86 \mathrm{~B}-4.28 \mathrm{AB}-3.08 \mathrm{~A}^{2}-2.63 \mathrm{~B}^{2}
\end{aligned}
$$

The final equation in terms of actual factors:

$$
\begin{aligned}
& \mathrm{EE}(\mathrm{R} 1)=6.18+340.53 \mathrm{~A}+14.97 \mathrm{~B}-25.25 \mathrm{AB}-354.50 \mathrm{~A}^{2}-1.17 \mathrm{~B}^{2} \\
& \mathrm{LT}(\mathrm{R} 2)=1.85+335.70 \mathrm{~A}+28.47 \mathrm{~B}-42.75 \mathrm{AB}-307.63 \mathrm{~A}^{2}-2.63 \mathrm{~B}^{2}
\end{aligned}
$$

The experimental results adequately fit the selected equation, which can obtain the response value of a random formula in the range of designed factors and levels by regression equations. To better comprehend the predictive models of the results, binomial model response surface analysis graphs were drawn (Fig. 1). With the increase in the ratio of PLs to the total excipients and total concentration of excipient, the EE and LT of G-Rg3 BS-PC-MMS changed. The optimal formulation of G-Rg3 micelles was screened by RSM; the molar ratio of egg yolk lecithin to total excipients was 0.40 , and the total concentration of excipient was $21.1 \mathrm{mg} \cdot \mathrm{mL}^{-1}$. Three batches of G-Rg3 BS-PC-MMS were prepared according to the optimized formulation. The EE and LT of obtained mi-

Table 2. Results of Central Composite Design

\begin{tabular}{crrrr}
\hline \hline Formulation (No.) & \multicolumn{1}{c}{$\mathrm{A}$} & $\mathrm{B}$ & $\mathrm{R} 1(\%)$ & $\mathrm{R} 2(\%)$ \\
\hline 1 & 0.000 & -1.414 & 87.5 & 92.3 \\
2 & 0.000 & 0.000 & 91.5 & 99.4 \\
3 & 0.000 & 0.000 & 90.4 & 98.9 \\
4 & -1.414 & 0.000 & 81.9 & 92.4 \\
5 & 0.000 & 1.414 & 88.8 & 95.7 \\
6 & -1.000 & 1.000 & 88.5 & 97.8 \\
7 & 0.000 & 0.000 & 91.1 & 99.5 \\
8 & 1.000 & 1.000 & 83.9 & 89.9 \\
9 & 0.000 & 0.000 & 91.9 & 99.5 \\
10 & 1.000 & -1.000 & 89.1 & 97.4 \\
11 & 0.000 & 0.000 & 89.9 & 98.9 \\
12 & -1.000 & -1.000 & 83.6 & 88.2 \\
13 & 1.414 & 0.000 & 84.9 & 93.8 \\
\hline
\end{tabular}

R1: Entrapment efficiency (EE); R2: light transmittance (LT). 


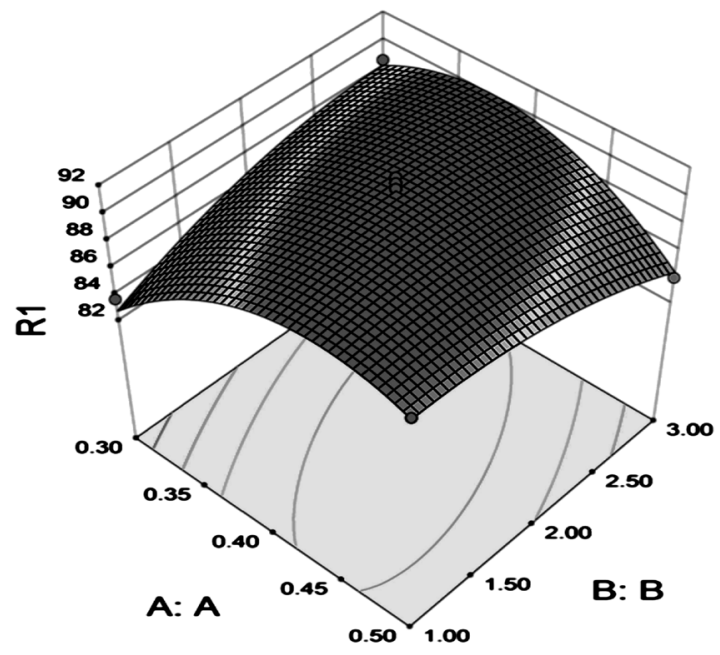

a

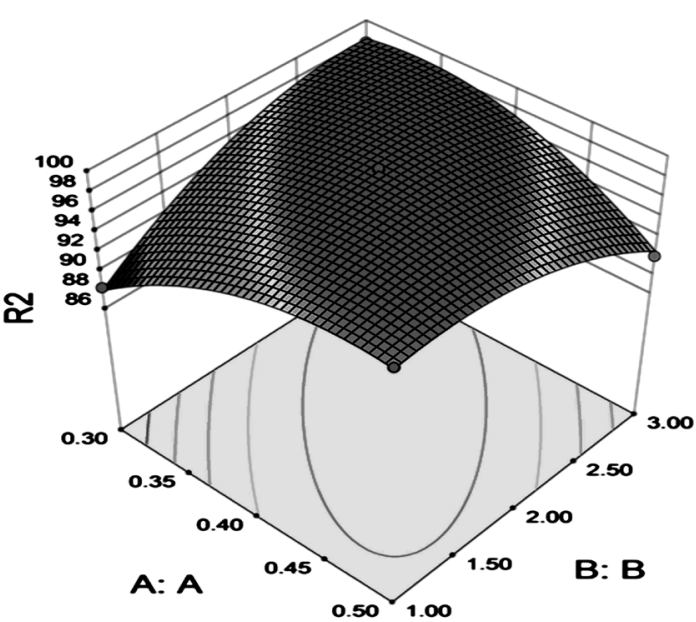

b

Fig. 1. Response Surface Plot Showing the Significant $(p<0.05)$ Interaction Effect of the Encapsulation Efficiency (R1) (a) and the Light Transmission (R2) (b) as a Function of Independent Variables

(A) $\mathrm{EPC} / \mathrm{EPC}+\mathrm{SDC}$ (molar ratio), (B) the total concentration of excipients (\%).

celles were $90.69 \pm 2.54 \%$ and and $99.10 \pm 3.12 \%$, respectively. The predicted EE $(90.72 \%)$ and LT $(99.20 \%)$ were in close agreement with the actual values. These results indicate that this experimental model was adequate and reproducible.

Numerous preliminary related experiments have been performed to investigate the nature of the two materials of micelles, namely, bile salts and PLs. For sodium cholate (SC) and SDC under the same conditions, fewer hydroxyl groups indicate greater lipophilicity and stronger BS-PC-MMS solubilization ability. It is well known that SDC has fewer hydroxyl groups in its structure than SC. Further experiments validated that SDC has a stronger solubilization ability to G-Rg3, so it was chosen as the material for micelles. The influence of PLs types on micelle formation was also compared. The results indicate that the stability and EE of micelles made of egg yolk lecithin were significantly better than those of soy lecithin.
The possible reasons for this result could be the lower content of PC in soy lecithin and more impurities and low amount of PC solubilized by SDC. Thus, high pure egg yolk lecithin exhibited a greater advantage, and the suitable content of PC in the PLs for preparing BS-PC-MMS should be greater than $95 \% .^{25)}$

Before the optimal prescription of BS-PC-MMS was determined by $\mathrm{CCD}$, univariate screening and uniform design approach were also performed for preliminary research. The results were not always satisfactory, and the error was relatively large. Therefore, these two methods were not adopted. However, the category of experimental factors and levels and the approximate range of the experimental center were determined. Thus, the pre-experiment had an important function in the successful implementation of CCD. Based on the preexperiment results of univariate screening, many factors could affect the EE and LT of G-Rg3 micelles. Among these factors, the molar ratio of bile salts to PLs and total concentration of excipient were the most important. The temperature and $\mathrm{pH}$ of the hydration medium had less effects on the EE and LT. Thus, $37^{\circ} \mathrm{C}$ and $\mathrm{pH} 7.4$ were selected to prepare micelles, which were relatively mild operating conditions and commonly adopted in the literature. ${ }^{25)} \mathrm{CCD}$ was conducted using the molar ratio of egg yolk lecithin to excipients and total concentration of excipient as the two investigation factors, and the other factors were fixed. The preparation of G-Rg3 micelles was optimized. The increase in proportion of PLs could improve the EE. In addition, the PLs could inhibit and decrease the hemolysis and cytotoxicity of bile salt based on the literature. $^{31-33)}$ Therefore, the addition of PLs into mixed micelles is necessary. The increase in the total concentration of excipients also increased the EE of Rg3 micelles. By contrast, the EE decreased when the total concentration of excipients increased to a certain amount, which was probably due to the micelle system reaching saturation.

The insoluble drug was encapsulated in bile salt and phospholipids (PLs) mixed micelles (BS-PC-MMS) to improve its solubility, and nearly $100 \%$ of LT indicates that PLs is completely dissolved in the bile salt as well as the mixed micelles forms. The decrease of LT shows that the solubilization of PLs by bile salt has already reached saturation. ${ }^{25)}$ In addition, the higher the LT, the smaller the particle size of the formulation. When the particle size was enough small, the particles are not easily phagocytized by mononuclear phagocyte system. Therefore the high LT is favorable. Indeed, compared with EE, LT take a weaker effect on micelles, therefore the LT was set as the second factor when screening prescription by using RSM.

RSM was simpler than the orthogonal experiment method, and is more comprehensive than uniform design. In addition, RSM has fewer number of trials, high test precision, and can be applied to multi-factor and multi-level tests. The biggest advantage of RSM is that it can predict the optimal conditions even though tests are not carried out. However, preliminary experiments are necessary to determine the range of each factor. In this study, RSM was selected to optimize the formulation of G-Rg3 BS-PC-MMS. The experimental values of EE and LT were in good agreement with the theoretical value. This optimization method provides a basis for the new DDS of G-Rg3.

Stability of G-Rg3 BS-PC-MMS The stability data of G-Rg3 BS-PC-MMS are shown in Fig. 2. The LT of G-Rg3 
micelles exhibited only a slight decrease in $7 \mathrm{~d}$. The EE of micelle was unchanged in $5 \mathrm{~d}$. However, the EE began to decrease significantly on the seventh day $(p<0.01)$, which shows

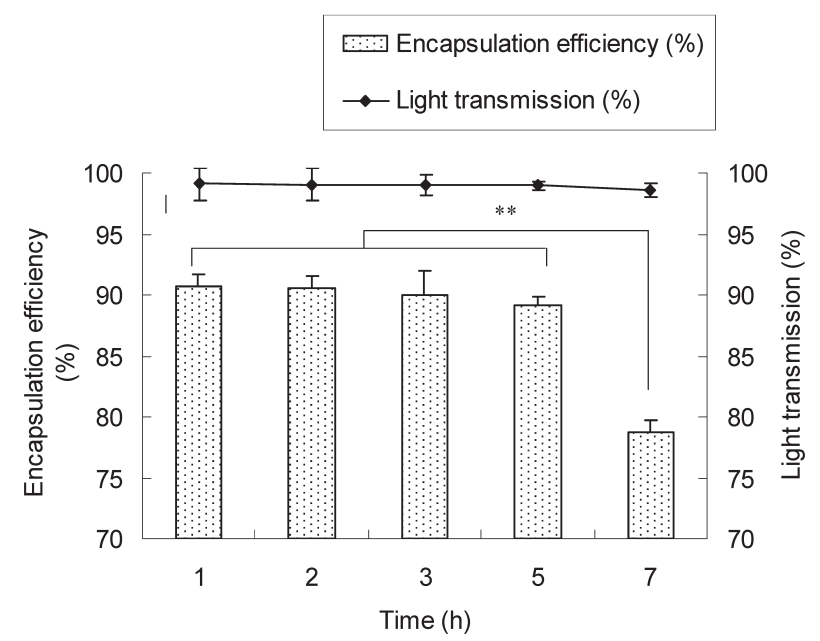

Fig. 2. Change in Encapsulation Efficiency and Light Transmittance of G-Rg3 BS-PC-MMS at Different Time Points

** represents $p<0.01$. that the stability of micelles decreased. Therefore, the liquid preparations of G-Rg3 BS-PC-MMS could be stored at $4^{\circ} \mathrm{C}$ for $5 \mathrm{~d}$. This short shelf-life could result in much inconvenience for the drug's application. Thus, G-Rg3 BS-PC-MMS were lyophilized in this study.

The stability of G-Rg3 BS-PC-MMS in vitro was relatively poor, so micelles were prepared in lyophilized formulation. Trehalose was selected as the lyoprotectant of micelles because it is a natural disaccharide with high stability and safety, and it also has anticorrosive function. The top groups of PLs in micelles can combine with the water molecules of external aqueous phase. Dehydration in the process of lyophilization may cause the destabilization of micelles and leakage of the drug. Many hydroxyl groups in the trehalose structure can replace the hydroxyl groups of water, and combine with the head groups of PLs and strengthen PLs membrane binding. Even after dehydration, membrane damage does not occur. ${ }^{33)}$ In the process of freeze drying, if the actual temperature of the system is higher than the glass transition temperature $\left(T_{\mathrm{g}}\right)$, the formulation will produce many adverse effects such as viscosity decrease, surface shrinkage, microscopic structural damage as well as drug leakage. $T_{\mathrm{g}}$ is one of important lyophilized formulation parameters and the temperature of the system should not exceed $T_{\mathrm{g}}$ of freeze-dried substance. Therefore

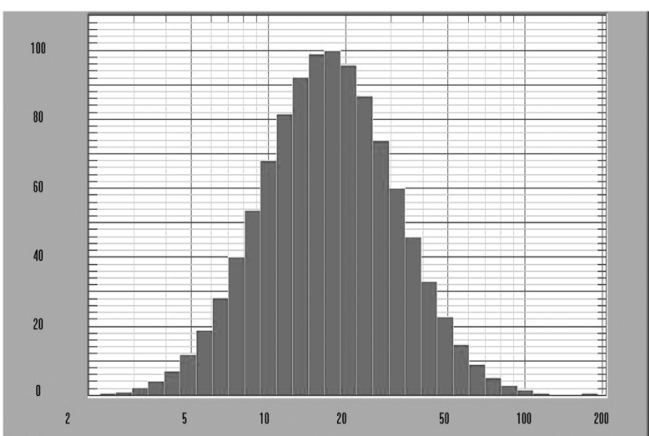

a

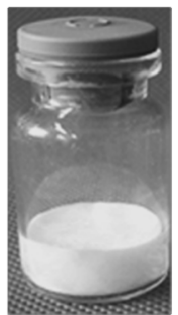

c

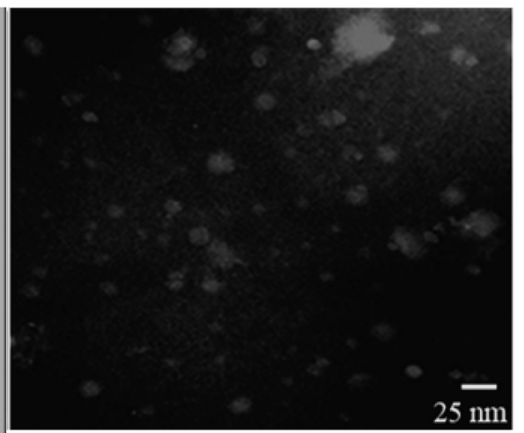

b

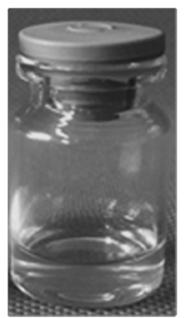

d

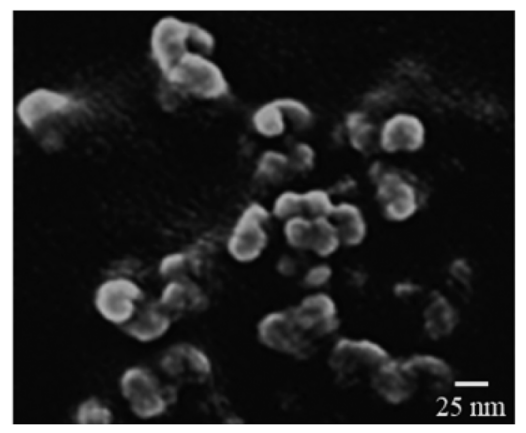

e

Fig. 3. Average Diameter Distributions (a), TEM Photograph (b) of G-Rg3 BS-PC-MMS, the Bar Represents $25 \mathrm{~nm}$; the Appearance (c), Reconstituted Preparation with Water (d), and SEM Photograph (e) of Lyophilized G-Rg3 BS-PC-MMS 
the drug leakage of liposomes or micelles in the process of freeze-drying can be prevented or slowed by increasing $T_{\mathrm{g}}$ of the system. ${ }^{34,35)}$ It is favorable to maintain the vitrification degree and increase the stability of preparation by choosing the higher $T_{\mathrm{g}}$ of materials. Compared with other common cryoprotectants, such as glucose, trehalose, sucrose, and maltose, trehalose has a higher $T_{\mathrm{g}}$, which could explain why trehalose possesses prominent protective effects on lyophilization. The final G-Rg3 lyophilized micelles had a smooth and delicate appearance and short reconstitution time compared with those of freshly prepared micelles. The particle size, EE, and LT of lyophilized micelles did not significantly change.

Characterization of G-Rg3 BS-PC-MMS As shown in Fig. 3a, the mean particle size of G-Rg3 BS-PC-MMS determined by a laser particle size analyzer was only $20.6 \mathrm{~nm}$, and the particle size distribution was relatively homogeneous. As shown in the TEM graph (Fig. 3b), the micelles appeared disk-shaped, and their particle size was about $20 \mathrm{~nm}$, which was consistent with the size of the measurement results. According to the preliminary screening results in this study, trehalose was selected as the lyoprotectant of micelles. Photographs of the lyophilized and reconstituted preparation are shown in Figs. 3c and d, respectively. The lyophilized G-Rg3 micelles had a delicate, plump, and smooth appearance (Fig. 3c), and the reconstituted micelles were colorless and transparent (Fig. 3d). The EE and LT of reconstituted micelles were $87.98 \pm 2.13 \%$ and $97.9 \pm 1.02 \%$, respectively, and these values were not different from those of freshly prepared micelles $(p>0.05)$. As shown in the SEM of lyophilized G-Rg3 BS-PC-MMS in Fig. 3e, the micelles were shaped as regular spheres with a particle size less than $25 \mathrm{~nm}$, demonstrating only a minimal increase compared with the freshly prepared micelles. The $\zeta$ potential of G-Rg3 BS-PC-MMS is $-28.13 \pm 1.34 \mathrm{mV}$.

Hemolysis Assay of G-Rg3 BS-PC-MMS The in vitro hemolysis experiment was used to evaluate the safety of G-Rg3 micelles in vivo. As shown in Table 3, the HR increased from 1.54 to $4.72 \%$ with the increase in BS-PC-MM volume. This observed HR was less than $5 \%$, which was consistent with the clinical requirements of liquid formulations. The degrees of hemolysis were observed by the naked eye, as shown in Fig. 4. No hemolysis was observed in different concentrations of G-Rg3 micelles.

Direct observation and spectrophotometry were adopted to study the hemolysis of G-Rg3 micelles. The direct observation method can directly record hemolysis via photography, which is highly intuitive and simple. The released hemoglobin from ruptured red blood cells has a certain absorption in the visible wavelength range. Thus, spectrophotometry can evaluate hemolysis and eliminate the subjective error of the conventional in vitro test tube method. Compared with erythrocytometry, the operation is relatively simple. Hemolysis results indicate that the HR was less than 5\% when the amount of BS-PCMMS was controlled within a certain range. This value was in accordance with the requirements for in vivo applications.

Suppression of Tumor Cell by G-Rg3 BS-PC-MMS MTT assay was used to evaluate the inhibition of tumor cells by G-Rg3 micelles, free drug, and blank micelles on A375 melanoma cells. As shown in Fig. 5, the blank micelle group did not inhibit the cell lines, and cell viability was nearly $100 \%$. For the G-Rg3 micelle and solution groups, cell viability decreased with the gradual increase in drug concentration. The cell inhibition action of micelles was slightly higher than that of the solution. The $\mathrm{IC}_{50}$ of G-Rg3 BS-PC-MM and solution for A375 melanoma cells were $119.7 \mu \mathrm{g} \cdot \mathrm{mL}^{-1}$ and $106.7 \mu \mathrm{g} \cdot \mathrm{mL}^{-1}$, respectively, and the difference was not statistically significant.

Experiments on tumor cell inhibition showed that the inhibitory action was slightly less than that of $\mathrm{G}-\mathrm{Rg} 3$ solution at low concentrations of G-Rg3 micelles. However, the difference was not significant. As the concentration increased, the difference between the micelle and solution groups gradually decreased. The results indicate that the encapsulation of micelles did not affect the inhibition of G-Rg3 on tumor cells, and the micelle and solution groups were inhibited in a concentration-dependent manner.

Inhibition of Angiogenesis of G-Rg3 BS-PC-MMS The anti-angiogenesis activity of G-Rg3 micelles was studied on a widely used CAM model. The two concentrations of G-Rg3 BS-PC-MMS were added to CAM for $3 \mathrm{~d}$ of treatment. As

Table 3. Results of Hemolytic Test in Vitro

\begin{tabular}{ccc}
\hline \hline Tube No. & Absorbance & Hemolysis rate $(\%)$ \\
\hline $1-3$ & 0.079 & 1.54 \\
$4-6$ & 0.095 & 2.39 \\
$7-9$ & 0.115 & 3.45 \\
$10-12$ & 0.121 & 3.77 \\
$13-15$ & 0.139 & 4.72 \\
$16-18$ & 0.050 & - \\
$19-21$ & 1.934 & - \\
\hline
\end{tabular}

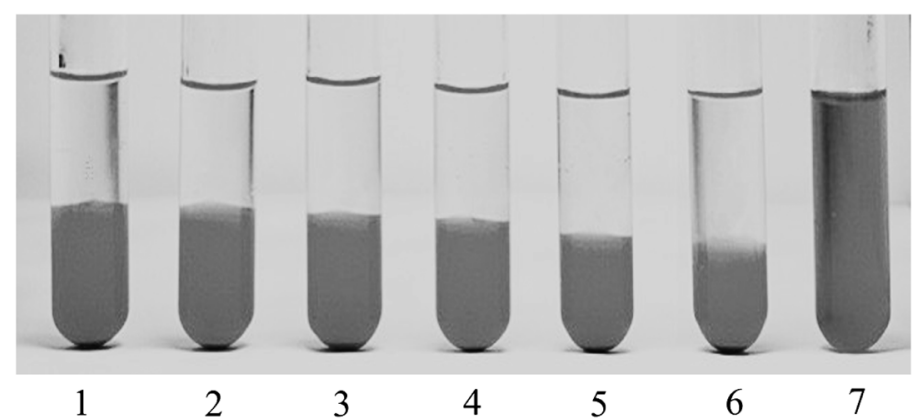

Fig. 4. Visual Inspection of the Tubes Containing Blood Samples Treated with Different Volume of G-Rg3 BS-PC-MMS

Nos. 1-5 are experimental group, the volume of G-Rg3 BS-PC-MMS are 0.06, 0.07, 0.08, 0.09 and $0.10 \mathrm{~mL}$, respectively; No. 6 is negative control group; No. 7 is positive control group. 


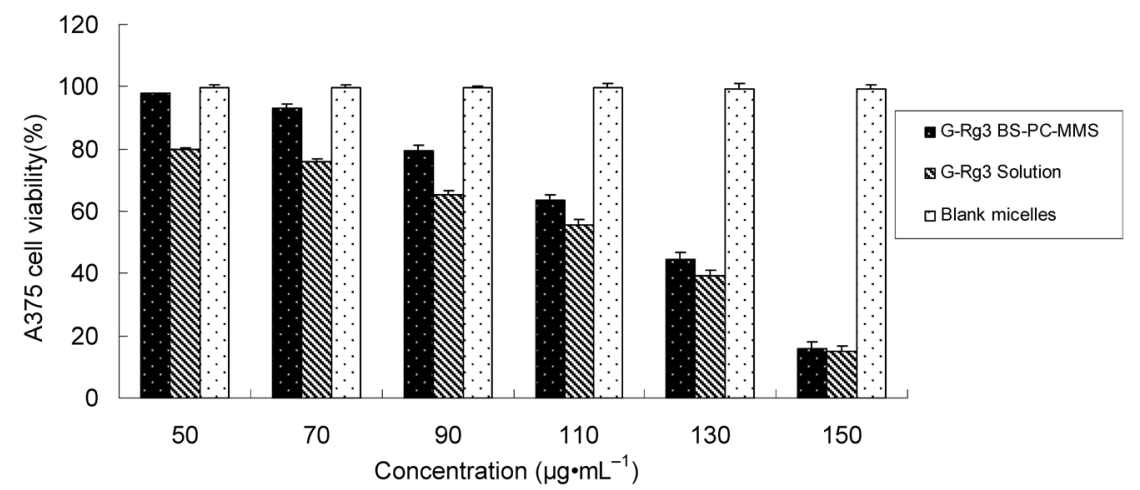

Fig. 5. A375 Melanoma Cell Growth Viability in the Presence of G-Rg3 BS-PC-MMS, Free Drug and Blank Micelles $(n=3)$

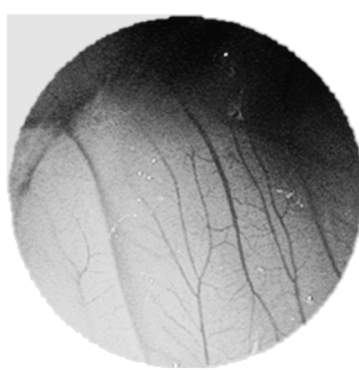

a

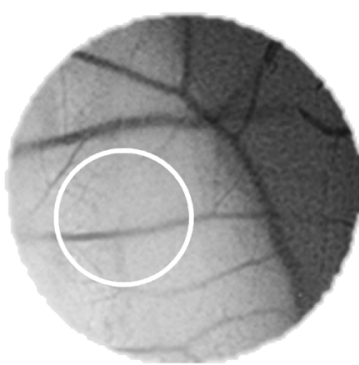

b

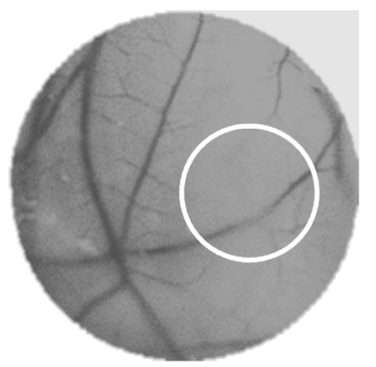

$\mathrm{C}$

Fig. 6. Effect of G-Rg3 BS-PC-MMS on Angiogenesis of CAM

a: Blank control group; b: G-Rg3 BS-PC-MMS (0.1 mM); c: G-Rg3 BS-PC-MMS (0.5 mM), white circle is the area of drug action.

shown in Fig. 6, compared with the control group, 0.1 and $0.5 \mathrm{~mm}$ of G-Rg3 micelles could significantly inhibit the angiogenesis of CAM. With the increase in the concentration of G-Rg3, the inhibitory effect improved and the density of blood vessels significantly decreased. When the concentration of G-Rg3 micelles increased to some degree, some chick embryos were unable to grow to the next developmental stage and died. No evidence of toxicity was observed in the chick embryos tested in all groups. These results showed that G-Rg3 BS-PC-MMS could significantly inhibit angiogenesis.

One of the targets of the anti-tumor effect of G-Rg3 is tumor angiogenesis. Abnormal angiogenesis is an important step in the development of solid tumors. ${ }^{36-39)}$ Therefore, anti-angiogenesis is a major means to control tumor growth. Anti-angiogenesis therapy has many advantages over other methods of cancer treatment. More than 40 well-known global pharmaceutical companies are developing anti-angiogenesis drugs, and at least 27 types of drugs have entered the clinical trial stage. ${ }^{40,41)} \mathrm{G}-\mathrm{Rg} 3$ has a multi-target effect on tumor cells, thereby affecting tumor angiogenesis. The inhibition of tumor angiogenesis by $\mathrm{G}-\mathrm{Rg} 3$ has been widely demonstrated. ${ }^{42-44)} \mathrm{In}$ this study, the chick embryo CAM assay was used to confirm the inhibition of neovascularization in G-Rg3 BS-PC-MMS. The chick fluff capsule has a rich vascular network and is sensitive to the inhibition of angiogenesis factors. In addition, the experimental operation of CAM is simple, and the results are directly viewed. The CAM is one of the best models for in vitro anti-angiogenic study. ${ }^{45)}$ The results show that G-Rg3 BS-PC-MMS could significantly inhibit tumor angiogenesis in a dose-dependent manner. When the concentration of G-Rg3 micelles increased to $0.5 \mathrm{~mm}$, all neovascularization disap- peared. G-Rg3 does not inhibit endothelial cell proliferation and has no cytotoxicity. Its anti-angiogenic effect may be related to regulated metabolism, which blocks the binding between endothelial cell membrane receptors and angiogenesis factor, and inhibits endothelial cell migration and other mechanisms. ${ }^{36,46)}$

\section{Conclusion}

In this study, G-Rg3 BS-PC-MMS were successfully prepared. The preparation process was very simple, and the obtained micelles had a high EE and small particle size. The micelles could significantly inhibit angiogenesis in the chick chorionic epithelium, had a strong inhibitory effect in tumor cells, and did not produce hemolysis in erythrocytes. Thus, this study preliminary achieved the desired objective. By contrast with the original oral formulation of G-Rg3, this new formulation of G-Rg3 micelles will significantly improve the efficacy, and reflect the obvious superiority of micelles as drug carriers in cancer treatment.

Acknowledgments This work was supported by $\mathrm{Na}$ tional Natural Science Foundation of China (81102394) and China Postdoctoral Science Foundation funded project (2013M530899).

Conflict of Interest The authors declare no conflict of interest.

Supplementary Materials The online version of this article contains supplementary materials. 


\section{References}

1) Oura H., Hiai S., Odaka Y., Yokozawa T., J. Biochem., 77, $1057-$ 1065 (1975).

2) Coon J. T., Ernst E., Drug Saf., 25, 323-344 (2002).

3) Pan X. Y., Guo H., Han J., Hao F., An Y., Xu Y., Xiaokaiti Y., Pan Y., Li J., Eur. J. Pharmacol., 683, 27-34 (2012).

4) Kim H. S., Lee E. H., Ko S. R., Choi K. J., Park J. H., Im D. S., Arch. Pharm. Res., 27, 429-435 (2004).

5) Lu P., Su W., Miao Z. H., Niu H. R., Liu J., Hua Q. L., Chin. J. Integr. Med., 14, 33-36 (2008)

6) Luo X., Wang C. Z., Chen J., Song W. X., Luo J., Tang N., He B. C., Kang Q., Wang Y., Du W., He T. C., Yuan C. S., Int. J. Oncol., 32 975-983 (2008).

7) Xu T. M., Cui M. H., Xin Y., Gu L. P., Jiang X., Su M. M., Wang D. D., Wang W. J., Chin. Med. J., 121, 1394-1397 (2008).

8) Xu T. M., Xin Y., Cui M. H., Jiang X., Gu L. P., Chin. Med. J., 120, 584-588 (2007).

9) Zhang Q., Kang X., Yang B., Wang J., Yang F., Cancer Biother. Radiopharm., 23, 647-654 (2008).

10) Carmeliet P., Jain R. K., Nature (London), 407, 249-257 (2000).

11) Folkman J., Semin. Cancer Biol., 3, 65-71 (1992).

12) Bergers G., Benjamin L. E., Nat. Rev. Cancer, 3, 401-410 (2003).

13) Jain R. K., Nat. Med., 7, 987-989 (2001).

14) Liu C. B., Zhang D., Li D. G., Jiang D., Chen X., Chem. Res. Chin. Univ., 24, 588-591 (2008).

15) Qian T., Cai Z., Wong R. N., Mak N. K., Jiang Z. H., J. Chromatogr. B Analyt. Technol. Biomed. Life Sci., 816, 223-232 (2005).

16) Sun C., Gao W., Zhao B., Cheng L., Anal. Chim. Acta, 492, 283293 (2003).

17) Cao F., Ouyang W., Wang Y., Chin. J. Chin. Mater. Med., 35 439-443 (2012).

18) Bie L., Yuan H. Y., Wang X., Zhao G., Liu X. J., Chem. Res. Chin. Univ., 26, 780-784 (2010).

19) Tsai W. C., Li W. C., Yin H. Y., Yu M. C., Wen H. W., Food Chem., 132, 744-751 (2012).

20) Yu H., Teng L., Meng Q., Li Y., Sun X., Lu J., Lee R. J., Teng L., Int. J. Pharm., 450, 250-258 (2013).

21) Sugioka H., Moroi Y., Biochim. Biophys. Acta, 1394, 99-110 (1998).

22) Hernell O., Staggers J. E., Carey M. C., Biochemistry, 29, 20412056 (1990).

23) Alkan-Onyuksel H., Ramakrishnan S., Chai H. B., Pezzuto J. M., Pharm. Res., 11, 206-212 (1994).

24) Alkan-Onyuksel H., Son K., Pharm. Res., 9, 1556-1562 (1992).

25) Hammad M. A., Müller B. W., Eur. J. Pharm. Biopharm., 46,
361-367 (1998).

26) Hammad M. A., Müller B. W., Eur. J. Pharm. Sci., 7, 49-55 (1998).

27) Mattila M. A. K., Suistomaa M., Anaesthesia, 39, 879-882 (1984).

28) Hammad M. A., Müller B. W., Drug Dev. Ind. Pharm., 25, 409-417 (1999).

29) Li Y., Yue W., Zou M. J., Xue X. B. M., Cheng G., Chin. J. Pharm., 4, 242-250 (2009).

30) Cheng X. B., Wang C. L., She Z. N., J. Shenyang. Pharm. Univ., 7, 497-503 (2012).

31) Narain P. K., DeMaria E. J., Heuman D. M., J. Surg. Res., 78, 131-136 (1998).

32) El-Hariri L. M., Marriott C., Martin G. P., Jpn. J. Pharmacol., 44, 651-654 (1992).

33) Tan Y., Qi J., Lu Y., Hu F., Yin Z., Wu W., Toxicol. In Vitro, 27, 714-720 (2013).

34) Crowe J. H., Crowe L. M., Chapman D., Science, 223, 701-703 (1984).

35) Janiak M. J., Small D. M., Shipley G. G., J. Biol. Chem., 254, 6068-6078 (1979).

36) Yue P. Y. K., Wong D. Y. L., Wu P. K., Leung P. Y., Mak N. K., Yeung H. W., Liu L., Cai Z., Jiang Z. H., Fan T. P., Wong R. N., Biochem. Pharmacol., 72, 437-445 (2006).

37) Fan T. P., Yeh J. C., Leung K. W., Yue P. Y., Wong R. N., Trends Pharmacol. Sci., 27, 297-309 (2006).

38) Folkman J., J. Natl. Cancer Inst., 82, 4-7 (1990).

39) Hori A., Sasada R., Matsutani E., Naito K., Sakura Y., Fujita T., Kozai Y., Cancer Res., 51, 6180-6184 (1991).

40) O'Reilly M. S., Holmgren L., Shing Y., Chen C., Rosenthal R. A., Moses M., Lane W. S., Cao Y., Sage E. H., Folkman J., Cell, 79, 315-328 (1994).

41) O’Reilly M. S., Boehm T., Shing Y., Fukai N., Vasios G., Lane W. S., Flynn E., Birkhead J. R., Olsen B. R., Folkman J., Cell, 88, 277-285 (1997).

42) Liu T. G., Huang Y., Cui D. D., Huang X. B., Mao S. H., Ji L. L., Song H. B., Yi C., Cancer, 9, 250-261 (2009).

43) Zhang Q., Kang X., Zhao W., Biochem. Biophys. Res., 342, 824-828 (2006).

44) Mochizuki M., Yoo Y. C., Matsuzawa K., Sato K., Saiki I., Tonooka S., Samukawa K., Azuma I., Biol. Pharm. Bull., 18, 1197-1202 (1995).

45) Nagaich S., Khopade A. J., Jain N. K., Pharm. Acta Helv., 73 227-236 (1999).

46) Chen M. W., Ni L., Zhao X. G., Niu X. Y., Chin. J. Chin. Mater. Med., 30, 357-360 (2005). 